\title{
Star formation history and isophotal shapes of fossil central galaxies
}

\author{
Habib G. Khosroshahi ${ }^{1} \dagger$ and Louisa A. Nolan ${ }^{1}$ \\ ${ }^{1}$ School of Physics and Astronomy, University of Birmingham, B15 2TT, UK \\ email: habib@star.sr.bham.ac.uk
}

\begin{abstract}
We study the structure and stellar populations of the luminous elliptical galaxies dominating fossil groups and compare them with the brightest galaxies in ordinary groups. Despite being over-luminous, the fossil central galaxies do not show boxy stellar isophotes which are usually associated with luminous elliptical galaxies. Boxy isophotes, according to the numerical simulations, are produced in gas poor mergers. The isophotal shapes of the fossil central galaxies, therefore, suggest a gas rich merger for fossil central galaxies. Using a two-component spectral fitting, we show that the dominant stellar population of the fossil and non-fossil galaxies is old and the second population is either old or intermediate age. However, the second stellar component (recently-formed stars) in fossil central galaxies is significantly more metal poor than that in the brightest galaxies of non-fossil groups.
\end{abstract}

Keywords. galaxies: evolution, galaxies: stellar content, galaxies: structure, galaxies: elliptical and lenticular, $\mathrm{cD}$

\section{Introduction and fossil groups}

It is believed that most of the large and luminous elliptical galaxies have formed via mergers of disk galaxies. This has been tested by the morphology-density relation and also extensively in computer simulations. The Brightest Group Galaxies (BGGs) are likely to have formed via mergers of galaxies within the group, though there is a high chance that the progenitors of the BGGs in early-formed groups are more gas-rich than the progenitors of the recently formed BGGs. There exists a class of galaxy groups known as "fossil groups" in which the group is dominated, optically, by a single luminous elliptical galaxy at the centre of an extended luminous X-ray emission similar to that seen in bright X-ray groups. The observed properties of fossils, and their absence of $L^{\star}$ galaxies, suggest that they must be old galaxy groups. These properties are discussed in a recent study (Khosroshahi, Ponman \& Jones 2007) where we report a relaxed and symmetric $\mathrm{X}$-ray morphology and a high dark matter concentration which are consistent with an early formation epoch for fossils and absence of recent major infall.

\section{Isophotal shapes of fossil central galaxies}

We study the isophotal shapes of 7 brightest galaxies in fossil groups using their deep R-band and K-band ground based images. This analysis shows that, despite apparent similarities, the dominant giant elliptical galaxy in fossil groups are different in their isophotal shapes, compared to the brightest central galaxies in non-fossil systems, especially in rich clusters. Luminous elliptical galaxies in non-fossil groups and clusters do not present disky isophotes. Less luminous BGGs show disky and boxy isophotes in similar

$\dagger$ Present address: Astrophysics Research Institute, Liverpool John Moores University, Twelve Quays House, Egerton Wharf, Birkenhead CH41 1LD, UK 
Table 1. Mass-weighted mean values for the best-fitting star-formation history parameters for the fossil and brightest group galaxy samples. The older population is labelled 1, and the younger, 2 . The uncertainties quoted are the standard deviations from the mass-weighted mean (Khosroshahi \& Nolan 2007). The Kolmogorov-Smirnov probabilities that the two samples are drawn from the same populations are also given.

\begin{tabular}{lccccc}
\hline & $<T_{1}>$ & $\left\langle T_{2}>\right.$ & $\left\langle Z_{1}>\right.$ & $\left.<Z_{2}\right\rangle$ & $<M_{1}^{\star} / M_{\text {tot }}^{\star}>$ \\
& $\mathrm{Gyr}$ & $\mathrm{Gyr}$ & $Z_{\odot}$ & $Z_{\odot}$ & \\
\hline BGGs & $13.7 \pm 0.7$ & $9.8 \pm 3.6$ & $2.03 \pm 0.68$ & $1.10 \pm 0.91$ & $0.79 \pm 0.18$ \\
Fossils & $14.0 \pm 0.0$ & $10.3 \pm 3.4$ & $1.74 \pm 0.75$ & $0.33 \pm 0.11$ & $0.67 \pm 0.19$ \\
KS probability & $(1-2.4 \mathrm{e}-7)$ & $(1-6.3 \mathrm{e}-5)$ & 0.932 & 0.193 & 0.871 \\
\hline
\end{tabular}

proportions. However, the observed frequency of boxy-isophote dominant fossil galaxies is apparently zero. According to numerical simulations (Khochfar \& Burkert 2005), disky isophotes are produced in gas rich mergers while the boxy isophotes are produced in dry mergers, though the mass ratios of the progenitors also seem to play a role in shaping the galaxy isophotes.

\section{Star formation history of fossil central galaxies}

We use a powerful long-baseline (UV-optical), multi-component, stellar population fitting technique (Nolan et al. 2007) to disentangle the star-formation history of the dominant central giant elliptical galaxy in a sample of five fossil groups, for which the spectrum was available, and compare this with a control sample of seven non-fossil brightest group galaxies, in order to understand their merger histories. Star formation activities are expected to have different histories depending on the mass ratios and the gas contents of the progenitors. The brightest group galaxies (BGG) sample was selected from the wellstudied groups in the Group Evolution Multi-wavelength Study, GEMS (Khosroshahi et al. 2004). Table 1 summarises the results of the spectral fitting.

\section{Conclusions}

We find that the old and the young stellar components in both galaxy samples are statistically indistinguishable in terms of their ages, however, the central fossil galaxies have secondary stellar components which have significantly lower metallicities than the corresponding stellar populations in the non-fossils. This combined with the non-boxy isophotal shapes of fossil central galaxies indicates a significant difference in the evolutionary history of the fossil and non-fossil central galaxies and it is consistent with the early formation epoch for the fossil systems and their dominant galaxies as there should have been more un-enriched gas available at the early epochs of the formation.

\section{References}

Khochfar S. \& Burkert A., 2005, MNRAS, 359, 1379

Khosroshahi H. G., et al., 2004, MNRAS, 349, 527

Khosroshahi H. G., et al., 2006, MNRAS Letters, 372, 68

Khosroshahi H. G., Ponman T. J., \& Jones L. R., 2007, MNRAS, 377, 595

Khosroshahi H. G. \& Nolan L. A., 2007, in preparation

Nolan L. A., et al., 2007, MNRAS, 375, 371 